\title{
Las pizarras digitales y su impacto didáctico en la educación superior
}

\author{
Digital whiteboards and their didactic impact in higher education.
}

\author{
Efraín Velasteguí López. ${ }^{1}$
}

\section{Resumen.}

En la actualidad nos encontramos donde las tecnologías invaden nuestros ámbitos de vida, que ha realizado muchas funciones de gran ayuda al entorno académico, funciones diarias, empresariales, en nuestra vida cotidiana. Las pizarras digitales ayudan a procesar la enseñanza aprendizaje en las instituciones educativas, estas herramientas tienen una gran variedad de potencial que se debe aprovechar para mejorar la enseñanza aprendizaje de los estudiantes haciendo las clases más didácticas e innovadoras con la ayuda del internet. La pizarra digital interactiva (PDI) es una alternativa nueva e innovadora en el ámbito educativo, es una herramienta que tiene un recurso importante para ayudar al aprovechamiento en las aulas tanto como al docente, como a los estudiantes de esta manera ambos aprovechan nuevos conocimientos de diferente manera ya que las pizarras digitales permiten realizar actividades como mapas conceptuales, exposiciones videos, consulta sobre alguna duda que tenga los estudiantes o el docente y de esta manera cambien la rutina de enseñar a través de dinámicas imágenes, para que haya una interacción por los estudiantes, realicen prácticas para adquirir de mejor manera nuevos conocimientos y les quede claro de que se trata el tema, porque la motivación del estudiante ayuda a mejorar su aprendizaje, ya que se han hechos estudios estadísticos a nivel mundial que tanto para los docentes como para los estudiante es de gran ayuda las pizarras digital para el proceso enseñanza aprendizaje y facilita la interacción en el aula.

Palabras claves: Pizarra digital interactiva, tecnología, innovación, metodología.

\section{Abstract.}

At present we are where technologies invade our areas of life, which has performed many functions of great help to the academic environment, daily functions, business, in our daily lives. Digital whiteboards help to process teaching learning in educational institutions, these tools have a great variety of potential that should be used to improve the teaching of students making the classes more didactic and innovative with the help of the internet. The interactive digital board (PDI) is a new and innovative alternative in the

\footnotetext{
${ }^{1}$ Ciencia digital, Ambato, Ecuador, luisefrainvelastegui@ cienciadigital.org
} 
educational field, it is a tool that has an important resource to help the use in the classroom as well as the teacher, as the students in this way both take advantage of new knowledge of different way since digital whiteboards allow to carry out activities such as conceptual maps, video exhibitions, consultation about any doubts that the students or the teacher have and in this way change the routine of teaching through dynamic images, so that there is an interaction by the students, do practices to acquire new knowledge in a better way and it is clear to them that the subject is treated, because the motivation of the student helps to improve their learning, since statistical studies have been made worldwide for both teachers and the student is a great help the digital whiteboards for the teaching and learning process easy ita interaction in the classroom.

Keywords: Interactive digital whiteboard, technology, innovation, methodology.

\section{Introducción}

Según la investigación de (Murado, J. 2012; \& Hernández, J. 2011) Las pizarras digitales es una de las innovaciones en las nuevas tecnologías que han aparecido en los últimos tiempos para apoyar la asistencia pedagógica en las aulas, para muchos maestros una pizarra digital interactiva no es más que una herramienta para utilizar en clases, aunque en realidad su potencial permite transformarlo todo desde la forma en que el profesor imparte la clase. Las pizarras digitales hayan ido evolucionando progresivamente, aunque manteniendo su esencia intacta, se trata de una pantalla que permite la interactividad que tiene un tamaño variable y desde la que es posible manejar y gestionara un ordenador aporta el componente digital. La tecnología que lo hace funcionar está muy presente en la vida de las personas, pero pasa casi desapercibida. Es necesario reconocer la evolución e la tecnología pues ha ido trascendiendo, desde su inicio con la pizarra blanca (1980) y el uso de proyectores de video (1990), y progresivamente el uso del internet a finales e inicios XXI, por lo cual a través de la historia se ha conocido desde los años 90 la presencia de estos recursos.

Las pantallas digital fomenta interactividad con su forma tan fácil de usar de manera que todos con verlas podrán manejar bien y, ya que el estudiante tiene forma de aprender de manera diferente e interactiva asiendo las clases divertidas y fácil de atender de esa forma atienden mejor sin aburrirse y con mucho mejor conocimiento para aprender ya que se puede ver cualquier duda del estudiante por medio del internet ya que todos los estudiantes no tienen la misma capacidad de aprender ni de captar conocimientos rápidos por lo que la pantalla digital nos ayuda tanto a los estudiantes como a los docentes a satisfacer todas las dudas planteas en la clase.

$\mathrm{Su}$ funcionamiento es fácil de usar ya que solo es de observar las cosas de nuestro alrededor y no podemos dar cuenta que no es nada complicado, ya que se puede manejar con los dedos, sin ninguna complicación es cómo manejar tu propio celular o cualesquiera otras cosas o se puede manejar con cualquier herramienta no puntiaguda ya que si se usa marcadores se puede dañar y sería muy costoso.

La pizarra digital mejora el proceso de enseñanza aprendizaje en los estudiantes como maestros ya que todos aprenden de nuevos conocimientos, y de manera fácil y eficaz de aprender, hacen que las clases se vuelvan más interesantes de la ayuda del internet se 
convierte una fuente de información y de gran ayuda para reforzar los conocimientos facilita la creación de enlaces de unión como los blogs, videos, etc.

En la sociedad actual el uso de las TIC ha logrado un gran conocimiento en nuestras vidas, para poder adaptarse en el futuro no solo porque las pizarras digitales aportan no solo a su uso básico, sino que se pueden hacer diversas actividades aplicaciones informáticas, exposiciones, trabajos, dudas, etc. Ayuda a los docentes integrarse de manera más fácil con sus proyectos y habilidades en las cuales se preparan como profesional y así se ayudan mutuamente y crecen no solo como profesional si no como persona estas pantallas digitales son creadas para apoyar las necesidades de los estudiantes ayudándoles a un mejor rendimiento académico.

Las pizarras digitales permiten una progresiva innovación entre los docentes y estudiantes por medio de prácticas, dudas planteadas por los estudiantes y le ayuda a mejorar la atención a las típicas clases comunes con la ayuda de herramientas que facilitan la enseñanza aprendizaje del docente como los estudiantes.

\section{Tecnologías y docencia}

Según los investigadores (Cerillo, A. 2010; \& Sancho, J. 2005) afirman que la adaptación de los modelos de aprendizaje a las nuevas tecnologías es un hecho que se demuestras en día a día de las aulas presenciales. En los últimos años se ha venido sustituyendo la pizarra tradicional por pizarras digital en muchas de las universidades públicas y privadas de nuestro país, Las aulas induce a una progresiva innovación de las practicas docenes a la vez que facilite una mejora del aprendizaje del estudiante, además el acceso a una fuente inagotable de información multimedia e interactiva disponible de manera inmediata en el aula, que permite aprovechar didácticamente muchos materiales realizados por profesores alumnos y personas ajenas al mundo educativo.

En estos tiempos, las actualizaciones de los docentes son primordial. La competencia digital consiste en disponer de habilidades para buscar, obtener, procesar y comunicar la información hasta su transmisión en distintos soportes una vez tratada, incluyendo la utilización de las tecnologías de la información y la comunicación como elemento esencial para informarse, aprender y comunicarse. Cuando los profesores adquieren el conocimiento y las habilidades suficientes en el uso de las pantallas digitales interactivas (PDI), son capaces de incrementar la interactividad e implementar una variedad de recursos, debido a que ella presenta herramientas que mejora considerablemente los procesos de enseñanza aprendizaje. Porque cada vez más la tecnología avanza a pasos agigantados. El reto que nos plantea la incorporación de la tecnología en el ámbito educativo conlleva que las recibamos con interés y receptividad, para lo cual las actitudes que mostremos son imprescindibles. De hecho, al analizar la integración de las TIC en los procesos educativos hay que considerar no solo las argumentaciones racionales sino también las emociones que hay detrás de ellas.

\section{De la sociedad tradicional a la sociedad de información}


De acuerdo con el sociólogo (Castells, M. 1996) Nos encontramos ante un nuevo modo de desarrollo de la sociedad que denomina sociedad informacional, la cual designa una organización social en la que la generación, el procesamiento y la transmisión de información se convierten en las fuentes fundamentales de la productividad y el poder, debido a las nuevas condiciones tecnológicas que surgen en este periodo histórico. Además, considera que entre las tecnologías de la información se incluyen las tecnologías de microelectrica, la información, las telecomunicaciones, etc.

Desde los inicios de la humanidad, se han desarrollado distintos avances y descubrimientos, que han ido dando origen a la aparición de diferentes tecnologías a lo largo de la historia. Estas nuevas tecnologías han logrado y facilitado el desarrollo de la sociedad, llenando a los seres humanos de herramientas para afrontar problemas que les iban surgiendo a medida del tiempo y que no tenían solución con los medios existentes en ese momento. En la actualidad como ya hemos indicado todas estas tecnologías están influyendo en la sociedad, se está instalando una nueva cultura y mentalidad, la rapidez con la que las nuevas tecnologías avanzan hacen que muchas veces los conocimientos se queden obsoletos y vayan surgiendo nuevos valores que provocan continuas transformaciones en todos los factores que afectan nuestras vidas. Todas estas nuevas tecnologías han tenido un impacto en todos los sectores de la sociedad, esto conlleva que poco a poco todo gire en torno a las TIC, proporcionando la aparición de nuevos sectores laborales, y desarrollando nuevas realidades sociales, se puede decir que las tecnologías se encuentran presente en la mayoría de actividades que las personas llevamos a cabo. La importancia de estas tecnologías no se queda en ser solo, una mera herramienta de comunicación o trabajo, sino que día a día de hoy se considera una de las acusas fundamentales del cambio estructural de la sociedad.

Se puede decir que la revolución tecnológica de la información está transformando los pilares del sistema social y se relaciona de manera directa con el surgimiento de nuevas formas de organización que afectan a todos los ámbitos, económicos, político, al modo de ver el mundo de pensar de comportarse siendo los medios de comunicación uno de los encargados de difundir estas nuevas tecnologías. Debido a la importancia de los medios en la sociedad estimo oportuno que estos sean considerados como tal en el sistema educativo porque constituye una parte esencial del medio en el que se está educando a los alumnos y porque en cierta manera su presencia obliga al ajuste de las demandas impuestas por las nuevas tecnologías con el fin de adquirir las competencias derivadas del uso de las tecnologías digitales. Nos damos cuenta que los medios de comunicación favorecen que las personas permanezcan en contacto con sus seres queridos o con el mundo entero para estar informados de lo que sucede en nuestro mundo y si lo vemos de otra manera la forma de manear todo esto es de manera sencilla, eficaz y atractiva por lo cual es un instrumento de gran ayuda para todos sin fijarnos en la edad. Para los alumnos como para toda persona es una necesidad esas nuevas tecnologías para desenvolverse sin problemas dentro de una sociedad que se ven nuevas maneras de ver la información y así cada quien va a utilizarla con sus beneficios correspondientes a la necesidad que requiera saber o adquirir. 
Figura 3. Aula tradicional versus aula digital

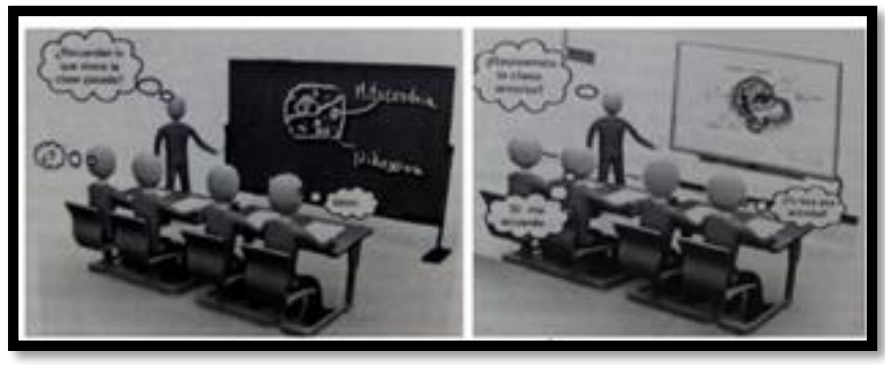

Fuente:http://espacio.uned.es/fez/eserv/tesisuned:EducacionMgomez/ GOMEZ_GOMEZ_Marta_tesis.pdf.

\section{La educación en la sociedad de la información}

Según la investigación de (Aguiar, V. 2002; \& Verde, M. 2014) La educación de la sociedad de información está caracterizada por la globalización económica tecnológica política y cultural que sea hablado de un paradigma tecnológico que tiene como información y transformación de la misma, esta revolución son efectos de la nueva tecnología en la mayoría de los ámbitos de la actividad humana con una incidencia tecnológica y cultural en el individuo como en la sociedad. La sociedad genera una cultura y esta cultura genera símbolos, signos y códigos que permiten comunicarnos día a día generando proceso de desarrollo. en la red de información se ha democratizado en el sentido de que está al alcance de un número cada vez mayor de personas de todos los niveles sociales con un fin distinto para satisfacer sus necesidades necesarias.

Los establecimientos educativos, debido a los avances tecnológicos que actualmente están cambiando el día a día de la sociedad, deben realizar un esfuerzo por introducir las nuevas tecnologías, para que de esta forma se pueda capacitar de una manera mejor a los alumnos en su utilización, lo que conlleva un cambio en los métodos de enseñanza aprendizaje. Se debe aprovechar el uso de esta tecnología, pero siempre teniendo en cuenta la importante labor que desempeña para los alumnos el profesor de manera que el uso de estas tecnologías nos ayude a mejorar y facilitar el proceso de enseñanza aprendizaje de los alumnos. El aprendizaje realizado en colaboración es una oportunidad interesante de desarrollo, no solo para estar informados sino también para comunicarnos con la sociedad, cuando este aprendizaje se nutre de tecnología como el ordenador, hablamos de aprendizaje colaborativo mediado por ordenador. Por ello, esta nueva forma de trabajar considera a las TIC como herramienta que permiten crear nuevas posibilidades de mediación social, nuevos entornos de aprendizaje y promover enlaces entre estudiantes y profesores, entre la propia comunidad educativa. La sociedad de la información debe convertirse en la sociedad del aprendizaje permanente. De esta manera, la formación y uso debe extenderse de las instituciones educativas convencionales había otros ámbitos. A su vez, es necesaria una alfabetización instrumental, critica y didáctico- metodológica. La formación a lo largo de la vida cobra especial importancia en un contexto de continuos cambios la formación inicial que reciben los maestros no siempre da respuestas a las necesidades actuales, por lo que la formación continua es clave. La educación en la 
sociedad en las tecnologías es mucha importancia ya que ayuda a los estudiantes a realizar su tarea llenarse de información necesaria y actual de los diferentes países no importa el lugar donde se encuentre, ya que día a día es importante estar actualizados para poder desenvolvernos en esta sociedad llena de tecnología y saber, durante los últimos tiempos la transformación de conocimientos ha causado como elemento principal la información oral y escrita, haciéndola cada vez más necesaria, a través de nuevas tecnologías las nuevas generaciones de alumnos nacen y crecen en una sociedad actualizada lo que ha dado un lugar importante en su vida cotidiana ayudando a sus conocimientos mentales, actitudes y emociones haciéndoles mejor personas con mayores conocimientos.

\section{La importancia de la integración de las tic dentro del aula.}

Los investigadores (Valverde, J. 2000; \& Domínguez, A 2006) El modelo educativo que se viene desarrollando en Extremadura desde el 2000, pone especial énfasis uno de los destacados es la importancia de las TIC el acceso a las tecnologías digitales (TD) como recurso educativo con lo que nuestra región se halla en condiciones favorables para incorporarse plenamente a la sociedad del conocimiento, se observan bastantes similitudes con relación al fenómeno de la integración de tecnologías digitales en el aula, una de las reformas necesarias en los sistemas educativos es la organización, el funcionamiento de las tecnologías digitales en los centros educativos o la acreditación del profesorado con competencia TIC se pone de manifiesto la necesidad imprescindible de un plan de política TIC desarrollado en cada centro educativo y adaptado a su concepción del proceso de enseñanza. Aprendizaje, sus necesidades educativas y sus recursos humanos.

En el proceso educativo de las TIC en el sistema educativo exige un cambio de paradigma que funcione a los centros educativos y al profesorado en el origen de la transformación y que, de paso a un modelo modular, descentralizado basado en necesidades reales, dinámicos y flexible. A lo largo de estos años se ha intentado llevar a cabo una integración de los ordenadores y de las nuevas tecnologías en los centros educativos, es un proceso que ha tenido diversas etapas y con múltiples factores de distinta naturaleza que han influido de una u otra manera dicha integración. Se empezó a entender que los efectos de las tecnologías sobre la enseñanza y el aprendizaje podría ser comprendido solamente si se analizaba como parte de la interacción de múltiples factores en el mundo complejo de los centros educativos, A partir de esto hemos llegado a una etapa en la actualidad en la que teniendo en cuenta todos estos factores que influyen en el ámbito educativo, se pretende para el aprovechamiento de las nuevas tecnologías y para la mejora del proceso de enseñanza aprendizaje que cada alumno tenga un ordenador con conexión a internet, además de ir incorporando otros recursos tecnológicos que ayuden a mejorar el proceso. Con la utilización de las TIC los alumnos se encuentran motivados, como consecuencia de estar motivados tienen más interés y dedican más tiempo a estudiar, por lo que aprenderán más estas nuevas tecnologías les atraen y hacen que presten más atención implicándose más en el trabajo. Los factores fundamentales que influyen en un verdadero aprovechamiento de estas múltiples posibilidades que ofrecen estas nuevas tecnologías, se trata del interés, ganas y la motivación que el profesor tenga de integrar y utilizar las 
TIC en el aula siendo esto un referente fundamental para el desarrollo de cualquier tipo de recurso que se utilice en el aula. Los docentes son fundamentales como elemento moderador de las TIC en el aula, en consonancia a esto, resultan muy importantes las actitudes que tengan en relación a las nuevas tecnologías. El profesor decide en qué posición mostrarse y el rol a tomar, lo que condicionara su trabajo y el aprendizaje de sus alumnos. Esto es así, porque los maestros son los principales responsables de involucrar a sus estudiantes en las tareas académicas y estimular aprendizaje. Por lo tanto, estos encuentran en la tecnología nuevas maneras de entretenerse, divertirse, comunicarse, informarse y también de formarse. Las pizarras digitales interactivas son un recurso de potencial eficaz y adecuado para el proceso de enseñanza aprendizaje.

\section{Beneficios específicos del uso de las pdi producen en el proceso de enseñanza aprendizaje}

Según la investigación de (Macías, M. 2017; \& Méndez, J. 2009) La disponibilidad de las PDI en las aulas, conlleva que el proceso de enseñanza y aprendizaje este sufriendo una renovación que se va extendiendo a todo el profesorado y a todas las materias, destacar que todos estos beneficios que se pueden dar con la utilización de este recurso, dependerán en gran medida de la actitud de los docentes ante las grandes posibilidades que se abre entre ellos con el uso de las PDI.

Cualquier profesor puede hacer uso de la pantalla digital interactiva (PDI) puesto que su utilización y manejo resulta muy sencilla, las presentaciones que se realiza a través de la PDI, ayuda a una mejor comprensión de determinados conceptos. Por lo tanto, desde un punto de vista objetivo, las posibilidades y funcionalidades que ofrece la pantalla digital interactiva (PDI) para conseguir motivar a los estudiantes, para reforzar y facilitar el aprendizaje o para mejorar el proceso de enseñanza del profesor son inmensas, debido promover la implicación de los docentes para no dejar pasar las múltiples oportunidades que ofrece. La participación de los alumnos durante la clase aumenta y se consigue una mayor interacción entre profesor y alumno, fomentando el debate de la materia, la implementación de las nuevas tecnologías en el ámbito educativo ha llevado a la aparición de nuevas actividades hacia los alumnos ya que es una herramienta que propone a las nuevas aulas digitales una nueva metodología enseñanza aprendizaje sobre la educación en centros educativos.

Figura 2. Inserción de documentos a la PDI.

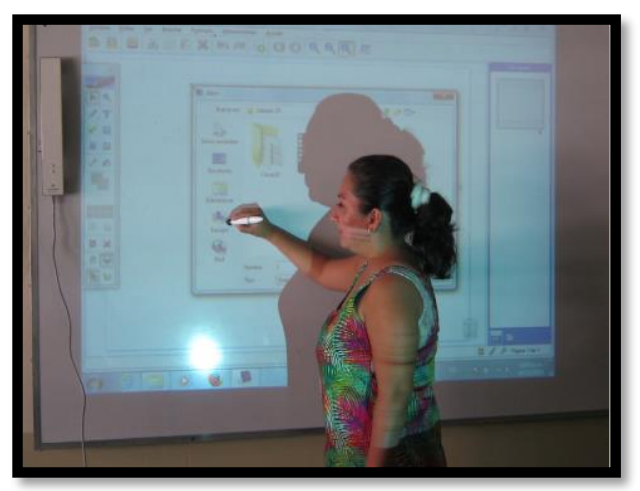


Fuente:http://e-spacio.uned.es/fez/eserv/tesisuned:Educacion

Mgomez/GOMEZ_GOMEZ_Marta_tesis.pdf.

\section{Modelos didácticos de la pdi en la educación}

Según (Medina, A. 2014) Teniendo en cuenta los múltiples beneficios que el uso de la pizarra digital interactiva nos puede aportar en el proceso de enseñanza aprendizaje en la educación, existen varias posibilidades de utilización y aprovechamiento de la PDI en el aula, entre ellas tenemos:

* Apoyo a las explicaciones del profesorado. Para que la comprensión de las explicaciones por parte de los alumnos mejore, el profesor puede hacer uso de diferentes materiales digitales como imágenes, esquemas, videos, no basándose únicamente en sus apuntes.

* Presentación de actividades y recursos para el tratamiento de la diversidad. Además, el uso de las PDI puede ayudar a que los alumnos que presenten algún problema de aprendizaje logren mediante la utilización de este recurso alcanzar los objetivos programados.

La sociedad en la que vivimos está evolucionando y experimentando una serie de cambios de diversa índole que nos obliga a replantearnos nuestra expectativas y funciones. Estos cambios precisan de una visión, misión y nuevas responsabilidades para adaptarnos a los mismos eficazmente. El uso continuo de las tecnologías ha supuesto un mayor acceso a la información, diferentes maneras de comunicarnos, de trabajar y también nuevas formas de enseñar y aprender. El paradigma educativo en el que este proceso se apoya es el constructivismo que favorece, entre otros aspectos, aprendizajes colaborativos y significativos. La presencia de las TIC en el ámbito educativo ya no es una novedad, sino una realidad, y en este entorno han llegado para quedarse y dar respuesta a las nuevas necesidades del aula y al nuevo perfil de alumno. Ante esta situación, debemos reflexionar sobre todo lo que implica, comenzar a replantearnos cosas, a aprender, quizás a desaprender. En esta cultura digital en la que estamos inmersos, los maestros deben utilizar las tecnologías no solo para alfabetizar digitalmente a los estudiantes, sino, sobre todo, para que sepan desenvolverse de manera efectiva y competente en el contexto en el que vivimos.

\section{Innovación e impacto de las tic en la educación}

De acuerdo a las investigaciones de (Vargas, M. 2006; \& Gacel, J. 2013) Las TIC en la educación se puede observar que sus primeros usos se centraron en el enfoque tecnológico. Con el paso del tiempo el énfasis cambia de la preocupación por lo técnico hacia el enfoque más humano y pedagógico las Tic en el aula ha generado una expectativa y ha despertado mucho interés y su uso y desarrollo. Las TIC construyen uno de los motores principales de innovación y desarrollo de un país, por lo que se deben dedicar los tiempos, recursos personales, materiales y económicos para su investigación. Sin embargo ¿que implica exactamente innovar? Este concepto más que un cambio externo 
requiere de una transformación interna en la manera de entender las cosas, los principios y fundamentos que dan sentido a la educación.

Con la innovación perseguimos mejorar una situación y se requiere de un componente ético qué de sentido al proceso, estamos en una época de grandes publicaciones, informes, investigaciones tanto internacionales como nacionales. Se está reflexionando cada vez más sobre el aprovechamiento del potencial educativo de las TIC, en la creación de nuevas metodologías de enseñanza y aprendizaje. Las tecnologías para el aprendizaje y el conocimiento (TAC) son, en realidad, el resultado de esta reflexión metodológica y, sobre todo educativa. En este sentido, se ha percibido cierta evolución desde la integración de las TIC hasta llegar a las TAC (integración con un sentido as pedagógico). Ya no nos conformamos con acceder y utilizar las herramientas de la web, sino que somos los protagonistas del proceso, se manifiesta en los próximos años las escuelas evolucionarán donde las redes sociales y el acceso a diferentes herramientas adquirirán un papel importante. En la dimensión psicosocial, las relaciones e interacciones en el espacio de aprendizaje total propician seguridad, confianza, autonomía y libertad en el alumno. Al mismo tiempo favorece la integración, comunicación y creación, generando una vivienda reflexiva a través de diversos recursos y lenguajes. Potencian la capacidad de asombro, el interés y la atención del estudiante.

\section{Estudios sobre la inclusión de la pdi en el aula}

Los investigadores (Pan, Z. 2008; \& Goig, R. 2014) afirman que los instrumentos tecnológicos aplicados a la educación, como es el caso de la PDI, son considerados hoy en día tecnologías emergentes que están introduciéndose en nuestras aulas y está modificando las maneras de enseñar y aprender. Esto no está ocurriendo únicamente en los centros educativos de países extranjeros, también es una realidad y un desafío para otros países.

La influencia es tan grande que la tecnología que mejor se ha integrado en los centros son, las versiones digitales de los recursos tradicionales son los libros digitales y pizarras digitales. La práctica educativa moldea el uso y la puesta en acción de la tecnología, la evolución y la convierte en parte indisociable de la práctica, los modelos de enseñanza más eficaces son aquellos que permiten al alumno activar sus conocimientos previos, adquirir y aplicar competencias básicas e integrarlas en el mundo real. En relación a la PDI ya vislumbrada su éxito al afirmar que, dentro de unos años, todas las aulas de todos los centros docentes tendrán una PDI al lado de la pizarra tradicional. Casi diez años después, aunque no todos los centros cuentan con esta tecnología las investigaciones realizadas hasta entonces confirman la gran coincidencia que esta y teniendo.

\section{Software de las pizarras digitales interactivas}

(Lamiña, K. 2014). expresa que en la sociedad podemos encontrar amplios manuales de software para pizarras interactivas orientados a la enseñanza aprendizaje y a elevar especialmente a elevar el aprendizaje en los educandos y facilitando la metodología y procesos educativos en el docente. A continuación, se citan algunos del software más 
utilizados, pero cabe enfatizar que los tipos y software de las pizarras varían acorde el diseño, modelo y marca de la pizarra digital que se adquiera.

\section{Smart}

Este es un tipo de sofware que posee multiples soluiones para la educación que se enfocan a la gestión del aula, el aprendizaje colaborativo hasta materias concreto para el desarrollo adecuado y óptimo de la clase.

\section{Eon Reality}

(Pizarra Interactivas, Sofware y Recursos Educación) en su blog de pizarras indica que: "Es un software interactivo para educación con el que crear y publicar contenido en formato 3D Multimedia. Esta solución en 3D ayuda a los alumnos a mejorar la comunicación y la transferencia de conocimientos mediante el aprendizaje basado en la simulación que ofrecen experiencias auténticas y realistas en cualquier momento y lugar".

\section{RM Easiteach Next Generation}

(GroupVision Distribution Services) en su blog de pizarras se expresa que: "Es el último software interactivo para el aula que se puede utilizar en cualquier pizarra digital, proyector y otros dispositivos de hardware, diseñado para ayudarle a crear y proporcionar lecciones motivadoras y atractivas. Easiteach permite a los usuarios crear lecciones personalizadas y recursos desde cero, así como acceder a una amplia gama de contenidos que pueden utilizar o adaptar para ajustarlos a sus propias necesidades".

\section{Interwrite Workspace}

"Interwriter Workspace es la nueva generación de programas para la enseñanza ideado para que un aula digital sea útil, efectiva y sobre todo sencilla para el profesor, tanto si es experto como novato en el uso de las nuevas tecnologías" (Interwriter). El software de Interwriter tiene incluido más de 4000 recursos especialmente dirigidos al área educativa, todos son de tipo digital y se encuentran a disposición del maestro o quien utilice la pizarra digital.

\section{El uso de las pizarras digital}

Según la investigación de (Gómez, M. 2015) El tratamiento de los datos obtenidos en la investigación es de vital importancia para el estudio. En el caso del análisis cuantitativo de datos a través de los cuestionarios se va a utilizar el Statistical Package for the Social Sciences (Paquete Estadístico para las Ciencias Sociales, SPSS), versión 22.0 para su tratamiento en relación a los niños de la etapa de educación primaria y, tal como se puede apreciar en la siguiente figura, a pesar de que todos los cursos están representados en la investigación, llama especial atención la elevada participación de los últimos cursos de cada ciclo en comparación a los primeros. Resaltando con mayor participación los niños de $6^{\circ}$ curso, seguidos de los de $2^{\circ}$ curso y los que están más presentes en el estudio son los de $4^{\circ}$ curso. Las edades más representadas son 7 y 9 años y la muestra se ha repartido equitativamente entre hombres $(51.4 \%)$ y mujeres $(48.6 \%)$. 
Figura 3. Participación en los niños de primaria



Fuente:http://e-spacio.uned.es/fez/eserv/tesisuned:Educacion Mgomez/GOMEZ_GOMEZ_Marta_tesis.pdf.

Tabla 1. El uso de las pantallas digitales interactivas (PDI) mejora las notas de los alumnos

\begin{tabular}{|ll|r|r|r|r|}
\hline & Frecuencia & Porcentaje & Porcentaje válido & Porcentaje acumulado \\
\hline Valido & Nunca & 127 & 16.4 & 16.4 & 16.4 \\
& Algrua vez & 220 & $\mathbf{2 8 . 4}$ & 28.4 & 44.7 \\
A menudo & 132 & 17.0 & 17.0 & 61.7 \\
Mucho & 288 & 37.1 & 37.1 & 98.8 \\
No sabe/ No contesta & 9 & 1.2 & 1.2 & 100.0 \\
Total & 776 & 100.0 & 100.0 & \\
\hline
\end{tabular}

Fuente:http://e-spacio.uned.es/fez/eserv/tesisuned:Educacion Mgomez/GOMEZ_GOMEZ_Marta_tesis.pdf.

(Gómez, M; 2015) Dado el carácter interactivo que lleva implícito la pizarra digital, nos parecía importante analizar la percepción de los alumnos en cuanto a la mejora de las relaciones e interacción con el profesor y entre los propios compañeros. Tal y como se aprecia en ambos gráficos la distribución de frecuencias es coincidente en ambas preguntas.

Figura 4. El uso de las pizarras digital interactivas (PDI) mejora la relación entre los alumnos 


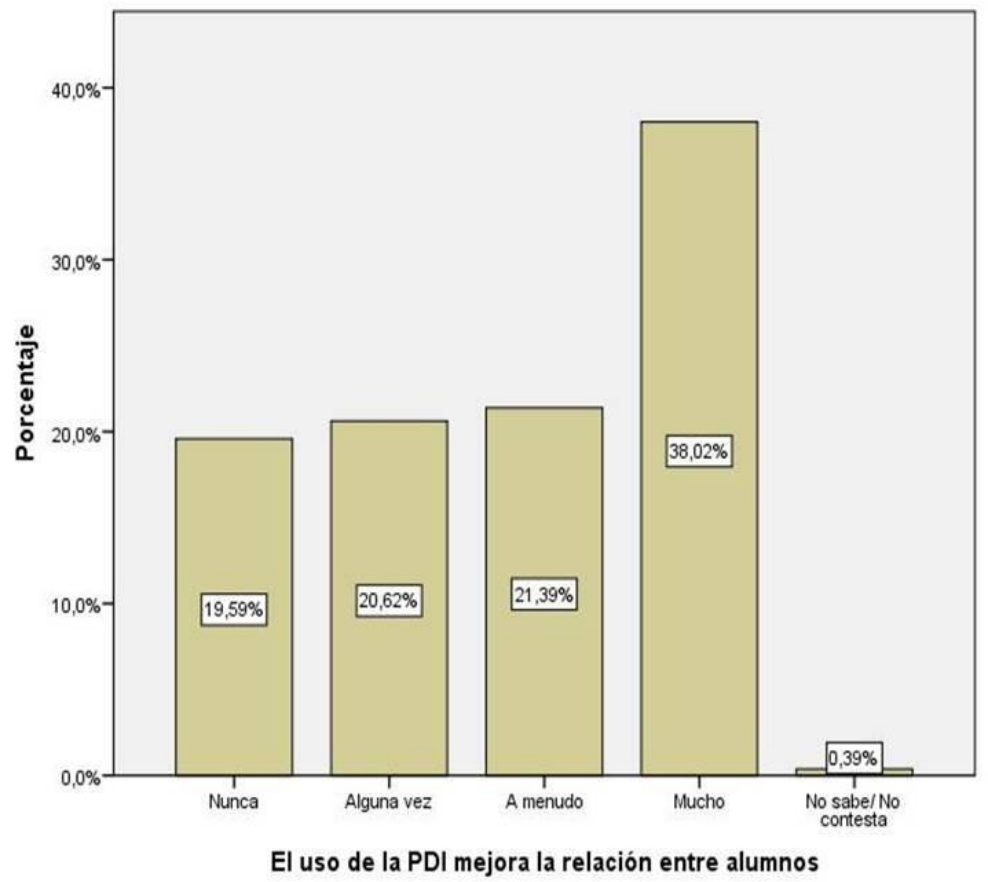

Fuente:http://e-spacio.uned.es/fez/eserv/tesisuned:Educacion Mgomez/GOMEZ_GOMEZ_Marta_tesis.pdf.

Ventajas y desventajas del uso de las pantallas digitales en el proceso enseñanza aprendizaje

Tabla 2. Ventajas y Desventajas de las pizarras digital

\begin{tabular}{|l|l|}
\hline Ventajas & Desventajas \\
\hline$>$ Permite que los estudiantes atiendan & $>$ El Docente debe poner de su parte para \\
con mayor énfasis sus clases. & $\begin{array}{l}\text { El Dodaptarse a las pizarras digitales. } \\
\text { más adecuada al tema. }\end{array}$ \\
Las pizarras digitales cuentan con el & $>\begin{array}{l}\text { Deben tener cuidado con las pizarras } \\
\text { acceso a internet. }\end{array}$ \\
$>\begin{array}{l}\text { Pueden salir de duda de algún tema } \\
\text { novedoso y poco entendible, ya que } \\
\text { todos los estudiantes no cuentan con la } \\
\text { misma retentiva. }\end{array}$ & $\begin{array}{l}\text { Son demasiadas cara que se debe hacer un } \\
\text { esfuerzo económico para conseguir una } \\
\text { para los centros educativos. }\end{array}$ \\
$\begin{array}{l}\text { Hacen las clases más didácticas e } \\
\text { innovadoras con la ayuda de esta } \\
\text { tecnología. }\end{array}$ & \\
\hline
\end{tabular}

Fuente: Elaboración propia

\section{Conclusiones}

La pantalla digital permite que los estudiantes adquieran conocimientos de manera que aumenta la motivación reforzando su interés y así las clases les resulta muy importante. 
> Las pantallas digitales ayudan a crear presentaciones dinámicas y eficaz que permiten que los estudiantes capten sobre la información brindada proporcionando la ayuda del internet para satisfacer sus dudas e inquietudes.

$>\mathrm{La}$ interacción que proporciona las pantallas digitales hacen que el docente interaccione con los estudiantes ayudándoles a reforzar sus conocimientos de manera de así debatir la clase.

$>$ Con las Pantallas digitales, se van aprendiendo cosas nuevas las clases dejan de ser aburridas tanto para los profesores como para los alumnos, de tal manera que se les hace divertida la clase y así atienden mejor ya que todos los estudiantes no tienen la misma retentiva, brinda una confianza mutua sobre los conocimientos que adquieran conocer.

\section{Referencias Bibliográficas}

Aguiar, V., \& Farray, J. (2002). Cultura y educacion en la sociedad de información. Netbiblo,S.L.. ISBN: 84-9745-027-2.

Castells, M. (1996). La era de la información economia social y cultura . siglo XXi editores. Argentina . ISBN: 968-23-2168-9.

Cerillo, A. (2010). Docencia del Derecho y tecnologías de la información y la comunicación. Barcelona . ISBN: 978-84-937606-5-6.

Domingez, A., \& Fernandez, M. (2006). Guía para la integración de las tics en el aula de idiomas. España. ISBN: 84-96373-99-1.

Gacel, J. (2013). Educación superior: Gestión, innovación e internalización España. ISBN: 978-84-370-9344-4.

Goig, R. (2014). Formación del profesorado en la sociedad digital. Investigación, innovación y recursos didacticos. Madrid. ISBN: 978-84-362-6891-1.

Gomez, M. (2015). La pizarra digital como recurso innovador y Favorecedor del proceso de enseñanza/aprendizaje De los alumnos de infantil y primaria del municipio De alcorcón. Madrid. Recuperado de: http://espacio.uned.es/fez/eserv/tesisuned:Educacion_Mgomez/GOMEZ_GOMEZ_M arta_Tesis.pdf

Hernández, J. (2011). Experiencias educativas en las aulas del siglo XXI. Madrid. ISBN: 978-84-08-10551-0.

Lamiña, K. (2014). La pizarra digital interactiva en el proceso de aprendizaje Del idioma inglés en los estudiantes de bachillerato de la Unidad educativa la inmaculada.Recuperado:http://www.dspace.uce.edu.ec/bitstream/25000/4134/1 /T-UCE-0010-583.pdf 
Macias, M., Zambrano, R., Intriago, J., Carpio, J., \& Marcillo, M. (2017). Methodological strategy for the right use of the interactive whiteboard in the teaching-learning process of the english language in the university context. Madrid. ISBN: 97884-947208-5-7.

Medina, A., De la Herrán, A., \& Dominguez, M. (2014). Fronteras en la investigación de la didáctica. Madrid. ISBN: 978-84-362-6833-1.

Mendez, J., Rodríguez, J., Dominguez, M., Lagándara, M., Olmos, S., Juanes, 1., Cabrero, J., \& Vasquez, R. (2009). Pizarras digitales interactivas multipunto: estrategia metodológica para una mayor particioacion y motivacion del laumno . Madrid. ISBN: 978-84-7800-208-1.

Murado, J. (2012). Pizarra digital. ISBN: 978-84-9839-420-7.

Pan, Z., \& Zhang, X. (2008). Technologies for E-Learning and Digital Entertainment. China. ISBN: 3-540-6973-4-9.

Sancho, J., (2005). Tecnologías para transformar la educación. España. ISBN: 84-4602486-1.

Vargas, M., Hernández, D., \& López, E. (2006). Divulgación y Video Científico. México. ISBN: 968-9024-05-1.

Valverde, J., Garrido, M.., Fernández, M., Soza, M., Revuelta, M., Arriazu, R., Rosado, F., Montero, J., \& López, C. (2004). Madrid. ISBN: 978-84-9085-255-2.

Verde, M., (2014). El impacto de las TIC en la sociedad actual. La pizarra digital. Recuperado de: https://uvadoc.uva.es/bitstream/10324/8103/1/TFGO\%20312.pdf. 


\section{Para citar el artículo indexado.}

Velasteguí E. (2019). Las pizarras digitales y su impacto didáctico en la educación superior. Revista electrónica Explorador Digital 3(1), 49-63. Recuperado desde: http://cienciadigital.org/revistacienciadigital2/index.php/exploradordigital/article/view/346/7 58

\section{Liencia}

El artículo que se publica es de exclusiva responsabilidad de los autores y no necesariamente reflejan el pensamiento de la Revista Explorador Digital.

El articulo queda en propiedad de la revista y, por tanto, su publicación parcial y/o total en otro medio tiene que ser autorizado por el director o editor de la Revista Explorador Digital.
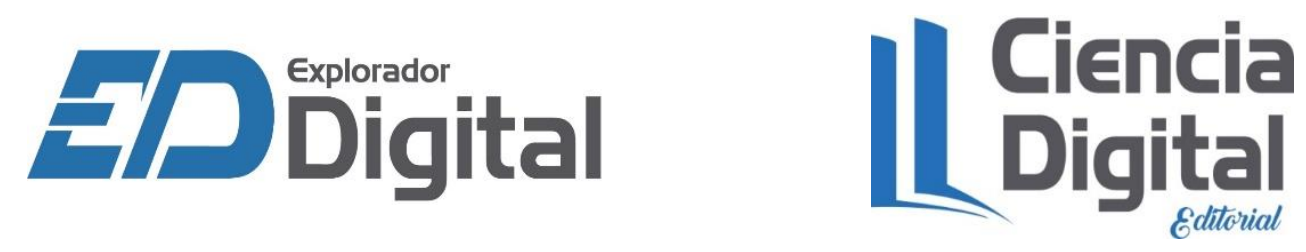